\title{
New/s Design: Informing Future Design Processes by Understanding Media Reporting of Medical Errors with Medical Devices [Invited paper]
}

\author{
Chrystie Myketiak \\ University of Brighton \\ College of Arts and Humanities \\ Falmer Campus \\ Brighton, UK BN1 9PH \\ c.myketiak@brighton.ac.uk
}

\author{
Shauna Concannon \\ Queen Mary University of London \\ School of Electronic Engineering and \\ Computer Science \\ London, UK E1 4NS \\ s.concannon@qmul.ac.uk
}

\author{
Paul Curzon \\ Queen Mary University of London \\ School of Electronic Engineering and \\ Computer Science \\ London, UK E1 4NS \\ p.curzon@qmul.ac.uk
}

\begin{abstract}
In this paper we present a case study of media reporting about medical technology issues. We discuss two incidents involving human error with medical devices that resulted in infant deaths, and their relevance for the medical device design and mobile health communities. Our analysis into the language and discourses of news reporting shows that the news narratives of these incidents emphasise human aspects of the error and neglect device issues. Better design is not conceptualised as an option in these narratives, even when systemic issues are discussed in relation to errors with devices. However, there is a possibility for better design solutions if practitioners are aware of the discursive construction of errors, including how critical incidents are framed and developed in news discourse.
\end{abstract}

\section{Categories and Subject Descriptors}

J.3 Life and Medical Sciences (e.g., Health)

J.5 Arts and Humanities (e.g., Linguistics)

\section{General Terms}

Design, Human Factors.

\section{Keywords}

Critical discourse analysis; human error; medical devices; news reporting; patient safety; sociolinguistics.

\section{INTRODUCTION}

The media helps drive technology cultures, yet its influence is underexplored within the medical device and mobile health communities. This study presents an analysis of news articles about incidents involving medical error and patient deaths. We examine the news reporting of two infant deaths in hospitals in different countries from the same temporal period using critical discourse analysis (CDA). Both deaths were linked to employee errors in administering medication using medical devices. Print and online news articles were gathered on these cases. We found differences in how the errors were discursively constructed. The first case involved an 'out by 10' mathematical error, which was often explained in the news articles as 'user error' with an interruption being the only systemic issue mentioned. The second case involved incorrect medication and the focus in that coverage was on workspace, personnel and medication placement changes that would be implemented in order to reduce similar errors in the future.

An important issue for the medical device design and mobile health communities is how, despite the differences in how these narratives frame the error in the news, there was no focus on the devices, their design, or use. Both cases lacked discussion of how poor design/usability could have contributed to the errors or of how improved design could prevent similar errors. Although there is a great deal of research on the issues and implementation of wireless sensors for health monitoring (e.g., [14, 27]), there has been little discussion of operator errors post-implementation. Our analysis suggests that when incidents with devices occur, the discussion in the media and the framing of investigations lacks emphasis on socio-technical systems and human-computer interaction (HCI) issues, including the use, design, and potential for improvement of device design. We assert that this propagates a discourse of technological determinism whereby individuals, organisations, designers, manufacturers, and the media may contribute to a narrative that users (individually or systematically) are responsible for errors and that thinks only of individual or ergonomic refinements rather than how use is impacted by good (and poor) design. This means there is little encouragement from these sources for error reduction improvements in designs and opportunities for designs to be iteratively improved are lost. 


\section{LITERATURE REVIEW}

The field of health communication is broad, with a long history of examining the dynamics of clinical conversations (e.g., [25]). More recently, there have been studies examining mediated interaction, such as emergency hotline calls (e.g., $[10,20])$ and health-oriented online communities (e.g., $[9,19])$. A complement to this research has been a focus on media representations of specific health topics (e.g., [4, 17, 18]). However, there has been little research on the intersection of media, health, and design.

We tie this body of work to that which focuses on design solutions in healthcare technology. In particular, patient safety is a serious technology concern (e.g., [11, 13, 23]). Issues surrounding technology and patient safety cover a wide terrain, including bridging strengths from many disciplines (e.g., computer science, engineering, mathematics, sociology, psychology, linguistics, etc.) to improve devices, clinical practices, and incident reporting.

The language of reporting cultures provides understanding of individual incidents. It also matters to healthcare technologists because it examines:

- the sociocultural environment of medical errors (e.g., real-life use as well as in the larger society where incidents are reported and stories are discussed)

- the linguistic and discursive construction of errors (e.g., whose story is told, where/how are users positioned)

- how the future of medical device design including mobile healthcare can be impacted by 'human error' incidents

\section{METHODOLOGY}

\subsection{Methods}

This study uses CDA, a qualitative methodology often used in sociolinguistics. We adopt corpus techniques as described by [1] to enable this. In addition to CDA, we provide supplementary quantitative data, as others using corpora approaches to discourse analysis in the area of health and illness have done [17, 18]. We concentrate on how these incidents are linguistically framed (cf. [21]); examining specific aspects of news discourse, including headlines $[2,7]$ and noun phrases [6].

The cases examined here were selected because they were critical incidents involving patient safety (infant deaths) and interactive medical devices (infusion pumps) in 2009 with sufficient English language news coverage for analysis. These two incidents are also comparative in that they took place in urban hospitals in Western countries (Canada and the United Kingdom) with publicly-funded health care.

- The first case ( $\mathrm{n}=80$ articles) involved a four-month old male infant in the UK who died two days after two nurses infused him with 10 times the prescribed amount of sodium chloride.

- The second case ( $\mathrm{n}=14$ articles) involved a six-week old male infant in Canada who died two weeks after pharmacists mixed Humulin R (insulin) rather than Heparin in his IV food (he was one of four premature babies who received the incorrect medication).

News articles were gathered through a combination of LexisNexis, which indexes news articles, and online searches in two countries (Canada and UK) in 2012 and 2013 to maximise the sample size; duplicates, such as wire articles running in multiple newspapers, are counted once.

The cases are referred to as Cases 1 and 2. We made a conscious choice not to use the names of the infants or staff who were involved in the incidents. While these names are part of the public record, we position this research as both sensitive and vulnerable [15] and wish to minimise further harm to those involved and their families. In addition to the infant deaths, nurses were blamed in one of the incidents; we do not want to contribute to a culture of 'naming, blaming, and shaming' [12] that some in the medical profession critique [24]. We maintain that this ethical decision does not impede our work.

\subsection{Data}

There was an inquest following the infant's death in Case 1, which involved a mathematical error whereby the patient received 10 times more medication than prescribed. Number entry research has shown that 'out by 10 ' are a standard class of errors that even skilled users may make [22]. This case received significantly more coverage than Case 2. The staff members involved in Case 1 were named in the coverage and the nurses were framed as culpable prior to the verdict. News reporting about Case 1 covered three distinct temporal periods $(2009,2010,2012)$.

In contrast with Case 1, no individuals were publicly blamed in Case 2, in which one of four infants who were infused with the incorrect medication on a neonatal ward died. The error originated in the hospital pharmacy when the incorrect drug was mixed into the patients' IV food. While the hospital acknowledged the error, a spokesperson said it was a 'mistake' and focused on contributing factors as well as procedural and spatial changes that would be implemented in the future.

\section{RESULTS AND DISCUSSION}

We focus our results and discussion on one central theme relating to the data of each incident and how its narrative is constructed. Following this we discuss the trend across the two incidents and their narrative arcs to neglect device-related components of the errors.

\section{1. $\quad$ Reading News Headlines}

Headlines draw readers' attention to news stories, and are critical to news discourse $[2,6,7]$. In examining the headlines associated with the cases where nurses were blamed for infant deaths (Case 1 ), the language used reduces the nurses to the incident and frames them in negative terms. More interesting is the variation in the headlines and how the language of headlines position nurses as incompetent through the deployment of lexical items.

Table 1. Case 1 Headline terminology analysis

\begin{tabular}{|c|c|c|c|}
\hline $\begin{array}{c}\text { Terms in } \\
\text { Headlines }\end{array}$ & $\begin{array}{c}\mathbf{2 0 0 9} \\
\mathbf{( n = 8 )}\end{array}$ & $\begin{array}{c}\mathbf{2 0 1 0} \\
\mathbf{( n = 2 4 )}\end{array}$ & $\begin{array}{c}\mathbf{2 0 1 2} \\
\mathbf{( n = 4 8 )}\end{array}$ \\
\hline $\begin{array}{c}\text { Nurse/s or name } \\
\text { Adjective applied } \\
\text { to nurse }\end{array}$ & 0 & 13 & 46 \\
\hline $\begin{array}{c}\text { Incident applied } \\
\text { as adjective to } \\
\text { nurse }\end{array}$ & 0 & 5 & 0 \\
\hline
\end{tabular}


Although two nurses were involved in the error, most headlines $(n=52)$ used the term 'nurse' or referred to one nurse by name, with an additional seven headlines using the plural 'nurses' (total 59/80 headlines). Further analysis also shows a temporal element: none of the articles from 2009 mention the nurse/s involved; instead all articles $(n=8)$ from that period focus on the patient. In 2010, the emphasis is evenly mixed with 24 articles, 13 of which have headlines referencing the nurses. However, in 2012, when the inquest into the death takes place, the headlines skew towards emphasising one nurse (45/48 headlines; an additional headline uses 'nurses'). Thus, there is a notable change over the news cycle of the story: while the subject of headlines immediately after the incident is the patient, in the two subsequent news cycles it is the nurse/s.

In the second reporting period (2010) there is also a discursive element of blame that occurs in some of the headlines that refer to the nurse/s. The use of adjectives works to frame the nurses as meriting blame. The five headlines that use adjectives to modify the nurse/s are all negative (lazy $(n=2)$, distracted, bungling, and sickest). The adjectival use terms such as 'bungling' or 'lazy' when preceding the term 'nurse' frame her as culpable for the error and challenge notions of her competency and work ethic. The use of the term 'sickest' in a headline frames the nurse in an exclusive category: first it is an absolute and second it implies that the error was purposeful rather than a mistake. Additionally, the focus on 'lazy', whilst not as dramatic, is a negative linguistic framing tool: it eliminates other possibilities that may have led to the error. Mathematical errors can occur for many reasons, individual and systemic, yet this immolates those options.

Other headlines reduce the nurse to the incident itself (e.g., 'saline baby death nurse', 'fatal blunder nurse' and 'salt-death nurse'). Although there was one of these found in 2010, as Table 1 indicates, the bulk of these $(n=9)$ were in the final reporting period (2012). In these examples, the death comes before the nurse, limiting her to it. The reduction of identity to the incident frames the nurse one-dimensionally as a 'salt-death nurse'. By using the incident to modify the noun, the nurse is reduced to the incident and, through the lack of person-first language, becomes defined by it.

\subsection{System-wide Responsibility in the News}

In Case 2 system-wide responsibility was narrativised in the telling of the incident, and received far less news coverage than Case 1. In more than half $(n=8)$ of the articles there was discussion of multiple spatial, ergonomic, and non-technical human factors that the hospital resolved to address in the future. Various other factors may also have contributed to Case 2 receiving less media attention: press culture and regulation differences within Canada affect what details can be reported and when [26]; and the decision not to publicly name any of the staff involved, resulted in a news story that did not have a 'villain' to anchor the narrative against a 'helpless victim', a pattern that can be found in news stories and other narrative forms, including folktales [5]. However, by immediately acknowledging systemwide factors and releasing a plan of how to avoid future errors of this kind, the hospital thereby engaged in 'image repair discourse' [3].

The hospital's public relations approach included addressing systemic factors that were not tied to individual behaviours (e.g., interruptions) but to space, staffing targets, and labeling practices. Action points included increasing the size of the hospital pharmacy and the number of pharmacists on staff; implementing safety checks, (e.g., having a second member of staff doublecheck medication); relabeling Humulin $\mathrm{R}$ to its generic name "insulin" in order to avoid potential confusion with similarly named drugs, such as Heparin; and storing insulin in a separate part of the pharmacy, away from other drugs, including Heparin. Of these action points the last two are specific to the incident changing how the drug is referred to and where it is stored - and will only prevent a like error from occurring in the future (i.e., an error involving insulin). So although these action points appear to broach system-wide issues that may have contributed to the error they do little in preventing other drug or medical device errors with similar causes. However, by addressing these concerns, the organization took control of the narrative, altering the story itself rather than the way it was framed and moved the focus away from individuals. What is critical is that regardless of the potential efficacy of these actionables with respect to patient safety, they are part of a narrative of 'taking responsibility' and allow the hospital to re-position the narrative. The hospital's own telling (through their spokesperson) of the incident and their proposed actions take the focus from the error and patient death and move it onto their response to the error.

\section{3. 'It's all about people'}

Donald Norman succinctly described good HCI as 'all about people' [16]. While people are critical in design processes, our analysis illustrates that devices and design are absent in this coverage. The errors in both cases were described as medication based: a 'medication error' and 'medication mix-up', respectively. In the total sample $(n=94)$ there was no discussion of the devices used, how the devices may have contributed to the errors, or how better design could have prevented errors. In sum, devices were rendered invisible: organisations and the media did not discuss the possibility that design and devices could be improved. The narratives expressed in the public domain were about people, who were constructed as victims and/or villains. This emphasis is different from what Norman and others have called for; the argument for design to be people-centred requires taking situated contexts and actual practices into account and embedding that knowledge into design from the earliest stages. A significant portion of the articles $(n=39)$ mentioned factors that contributed to the error (e.g., interruptions, physical space, labeling, double checking) that good design can broach; of those, 28 of the articles pertained to Case $1(\mathrm{n}=80)$ and focused on interruptions and the remaining 11 were from the Case 2 sample $(n=14)$, which covered a wider range of systemic factors. A small number of those articles also discussed changes in guidance that resulted from the errors ( $\mathrm{n}=3$ for Case $1, \mathrm{n}=8$ for Case 2 ), but again the focus was on what staff members could do differently or how the workspace could be re-organised. These media case studies illustrate a lack of discourse regarding better design or the possibilities of design: in public and work life technology and design are conceptualised as fixed rather than iterative.

\section{LIMITATIONS}

As this study consists of a CDA of two historic case studies and relies on the information in our corpus, we do not have detailed information about the specific device models used; however, through CDA, rich insights are garnered and issues surrounding error interpretation are highlighted that could be of particular relevance for the development of healthcare technology including mobile and remote healthcare application design. This paper offers a theoretical viewpoint that can enhance the understanding of error interpretation in health technologies and inform design protocols that encourage post-implementation, in situ evaluation and monitoring. 


\section{CONCLUSIONS}

While users are critical in usability and design processes, our research into news narratives illustrates that usability and design need to take a more prominent role in public discourse when errors occur. Our analysis shows that the reporting on usability related errors is already focused on people and their spaces and that this is problematic for HCI researchers and usability designers. Even when learning rather than blame is promoted, which has been encouraged by some (e.g., [24]) the discussion in these news narratives focuses on reframing user errors and workplace changes, including traditional ergonomics (e.g., moving supplies, making spaces larger), as is seen in Case 2. In both of our news media case studies, technology is seen as outside the realm of what can be transformed, and is discussed only in relation to 'training'. Therefore, the socio-technical system whereby these devices are used, and real-life errors actually occur may be neglected. This suggests a schism with how devices are presented and perceived: there is a focus on human factors in design processes and usability, yet when errors with medical devices occur the device is often rendered invisible. This occurs within the organisations where the devices are used as well as how those errors are communicated to the general public; this can be seen in how the organisations and the news frame the error. It is not simply that a wider understanding of human factor design issues is needed in the general population or within organisations. Rather, we propose that design itself may benefit from a more informed and reflexive outlook if HCI practitioners and medical device designers have both awareness and a better understanding of the culture of errors, including news reporting of critical incidents. If there were wider public discussion when medical incidents occurred that included the possibility of design changes preventing future errors, then this could promote innovation in design, rather than supporting the status quo.

\section{ACKNOWLEDGMENTS}

The authors gratefully acknowledge funding from the Engineering and Physical Sciences Research Council (UK), grant number (EP/ G059063/1).

\section{REFERENCES}

1. Baker, P. Using Corpora in Discourse Analysis. Continuum, London, 2006.

2. Bednarek, M. and Caple, H. News Discourse. Continuum, London, 2012.

3. Benoit, W.L. Image Repair Discourse and Crisis Communication. Public Relat Rev 23, 2 (1997), 177-186.

4. Berry, T.R., Wharf-Higgins, J., and Naylor, P.J. SARS Wars: An Examination of the Quantity and Construction of Health Information in the News Media. Health Comm 21, 1 (2007), 35-44.

5. Conboy, M. The Language of the News. Routledge, London, 2007.

6. Cotter C. News Talk. Cambridge University Press, Cambridge, UK, 2010.

7. Dor, D. On Newspaper Headlines as Relevance Optimizers. $J$ Pragmatics 35, 5 (2003), 695-721.

8. Eysenbach, G., Powell, J., Englesakis, M., Rizo, C., \& Stern, A. Health Related Virtual Communities and Electronic Support Groups: Systematic Review of the Effects of Online Peer to Peer Interactions. BMJ, 328, 7449, (2004), 1166.
9. Hamilton, H.E. Reported Speech and Survivor Identity in On-Line Bone Marrow Transplantation Narratives. $J$ Socioling 2, 1 (1998), 53-67.

10. Imbens-Bailey, A., and McCabe, A. The Discourse of Distress: A Narrative Analysis of Emergency Calls to 911. Lang Commun 20, 3 (2000), 275-296.

11. Kushniruk, A.W., Triola M.M., Borycki E.M., Stein B., Kannry J.L. Technology Induced Error and Usability: the Relationship Between Usability Problems and Prescription Errors when Using a Handheld Application. Intl J Med Inf 74, 7-8 (2005), 519-526.

12. Lester, H. and Tritter, J.Q. Medical Error: A Discussion of the Medical Construction of Error and Suggestions for Reforms for Medical Education to Decrease Error. Med Ed 35, 9 (2001), 855-861.

13. Lin, L., Vincente K.J., Doyle D.J. Patient Safety, Potential Adverse Drug Events, and Medical Device Design: A Human Factors Engineering Approach. J BioMed Info 34, 4 (2001), 274-284.

14. Milenković, A., Otto, C., \& Jovanov, E. (2006). Wireless sensor networks for personal health monitoring: Issues and an implementation. Comp comms, 29(13), 2521-2533.

15. Myketiak, C. and Curzon, P. Empathy and Medical Error Research Enabling Empathy. Health \& Care: Design Methods \& Challenges (CHI Workshop), (2014), 1-4.

16. Norman, D. The Design of Everyday Things. Basic Books, New York, 2002 [1988].

17. Seale, C. Sporting Cancer: Struggle Language in News Reports of People with Cancer. Sociol Health Ill 23, 3 (2001), 308-329.

18. Seale, C. Media Construction of Dying Alone. Soc Sci Med 58, 5 (2004), 967-974.

19. Stommel, W., Koole, T. The Online Support Group as a Community: A Micro-Analysis of the Interaction with a New Member. Discourse Stud 12, 3 (2010), 357-378.

20. Svennevig, J. On Being Heard in Emergency Calls. The Development of Hostility in a Fatal Emergency Call. $J$ Pragmatics 44, 11 (2012), 1393-1412.

21. Tannen, D., ed. Framing in Discourse. Oxford University Press, Oxford, 1993.

22. Thimbleby, H, Cairns P. Reducing Number Entry Errors: Solving a Widespread, Serious Problem. J. R. Soc. Interface 7, 1 (2010), 1429-1439.

23. Vincent, C, Blandford, A. Designing for Safety and Usability User-Centred Techniques in Medical Device Design Practice. Proc Hum Fact Erg Soc 55, 1 (2011), 793-797.

24. Waring, J.J. Beyond Blame: Cultural Barriers to Medical Incident Reporting. Soc Sci Med 60, 9 (2005), 1927-1935.

25. West, C. When the Doctor is a 'Lady': Power, Status and Gender in Physician-Patient Encounters. Symbolic Int 7, 1 (1984), 87-106.

26. Young, M.L., and Pritchard, D. Cross-Border Crime Stories: American Media, Canadian Law, and Murder in the Internet Age. Am Review of Canadian Studies 36, 3 (2006), 407-426.

27. Yan, H., Huo, H., Xu, Y., \& Gidlund, M. (2010). Wireless sensor network based E-health system: implementation and experimental results. IEEE $T$ Consum Electr, 56(4), 2288-2295. 